\title{
218 Juvenile respiratory papillomatosis
}

A Commonly involves the vocal cords and ventricular bands.

B Is primarily a disease of young adults.

C Should be treated with radiotherapy.

D May require laryngectomy.

E Is caused by a haemolytic positive streptococcus.

\section{Benign lesions of the larynx}

A Granular cell myoblastoma histologically shows profuse pseudo-epitheliomatous hyperplasia.

B Retention cysts occur most commonly in the ventricular sinus.

C Chondromata usually arise from the arytenoid cartilages.

D Benign tumours occur more frequently than malignant tumours.

E Adult haemangiomata usually require surgical excision. 